\title{
Survey paper on Context Sensing and Proximity Sensing for Smartphones
}

\author{
Arun Algude
}

\author{
Akshay Jain
}

Aditya Chaudhari

\author{
Rupali S. Vairagade
}

\begin{abstract}
Context awareness is obtaining more and more vital for variety of mobile and pervasive applications on these days smartphones. Whereas human centric contexts(e.g. indoor/outdoor, at homing/office, driving/walking) are extensively researched, few tries have studied from phones perspective(e.g. on table/sofa, in pocket/bag/hand). Thus everyone including us tend to ask such immediate surroundings as micro-environment typically many to a dozen of centimeters, around a phone. Styling and implementing good smart application, a micro-environment sensing platform that automatically records sensing data information and characterize the micro-environment of smartphones, is the main aim. The platform runs as a daemon process on a smartphone and provides finer-grained atmospheric information to upper layer applications via programming interfaces. Smart android application is a unified framework covering major cases of phone uses, placement, perspective and interaction in sensible uses with sophisticated user habits. As a protracted term running middleware, smart android applications consider both energy consumption and user friendly relationship.
\end{abstract}

\section{Keywords}

Pressure Sensor, Memory Management, Context Management, Process Management, Proximity Sensor

\section{INTRODUCTION}

Residing in the twenty first century, that is the century of technology and innovation, Smartphone is one, among the samples of innovation created during this century. These days Smartphone's having wide ranges of sensing computation and storage resources. There are several types of sensors as Proximity, Accelerometer, Camera, Touch, GPS etc. we tend to used these sensors associate degreed developed an automaton application. The idea of micro-environment sensing is constructed on each context sensing and contextawareness applications, nonetheless differs in its stress on perceiving immediate surroundings from the Smartphone's perspective. In mobile systems, context-awareness may be a computing technology that comes with data regarding the present surroundings of a mobile user to supply additional relevant services to the user. It is a key part of present or pervasive computing and has attracted several analysis efforts within the past decade. Most context-aware applications (via mobile sensing) are human-centric, recognizing contexts from users' perspective (e.g., indoor/outdoor, at home/in workplace, driving/walking. Such data supports services per users' state of affairs. This project consists of various modules like automatic decision picker, pressure sensing element used for security, GPS sensing element to trace the placement once wrong pattern entered, soft surface detection so as to activate the ringer mode, closed surroundings identification for battery saving purpose etc. So with all these features battery improvement is also been cared conjointly during this project to make the application more efficient.

\section{RELATED WORK}

In previous papers studied, [3] it is clearly seen that all of them targets application on a single sensor. They're creating one application by creating the employment of knowledge broadcasted by sensing elements. This application can consume a lot of battery because it must run unendingly. They need not created any adjunct application to save lots of the battery consumption. In this paper, applications or features are being developed which come under security and optimization domain. Smart Android App, a micro-environment sensing platform that automatically records sensor hints and characterizes the micro-environment of smart phones. The platform runs as a daemon process on a sensible phone and provides finer-grained setting data to higher layer applications via programming interfaces [5]. Smart android App could be a unified framework covering the foremost cases of phone usage, placement, attitude, and interaction in sensible uses with difficult user habits. As a semi-permanent running middleware[8]. Smart Android App considers both energy consumption and user friendship.

The sensors which are used in this application are Accelerometer, Vibrator, Touch Screen, Camera Gyroscope, Proximity etc. If a mobile phone is in a bag or pocket, it is useless to light up the screen when a phone call is coming. In addition, if a phone is placed on a sofa rather than on a desk, it is better to turn up ring volume to avoid missing calls.[8] Given accurate micro-environment information, a phone can adapt its behavior automatically and properly. To build the framework of Smart Android App upon associate investigation of phone usage and user habits will be the main aspect. The framework covers the bulk of phone's states, and consists of three core modules: phone placement detection so as to activate the ringer mode, closed setting identification for battery saving purpose and material detection for sensing the environment etc. Care of battery improvement has also been taken additionally during this project to create it a lot of economical. Smart Android App, a micro-environment sensing platform that mechanically records sensing element hints and characterizes the micro-environment of good phones. The platform runs as a daemon method on a sensible 
phone and provides finer-grained data to higher layer applications via programming interfaces

The framework covers the bulk of phones' states, and consists of three core modules: phone placement detection, phone interaction detection, and backing material detection. Phone placement refers to the situation of a smartphone beside its user, and considering the things in bag, in chest pocket, in pants, and in hand, whether or not a user is concentrating on his smartphone is another key judgment for microsurroundings sensing. At last, backing material detection analyzes the hardness of the things that touches (or holds) the phone.

In summary, the key contributions of this paper are: 1st, sensible Smart Android App may be a unified microenvironment sensing framework. though some previous works have enforced a part of similar practicality for easy environments, they can't be directly combined to an applicable level for sensible use with difficult phone things and user habits. Second, as a middleware run on sensible phones, sensible Smart Android App is each energy optimized and user friendly. Favoring to style a gradable design and a collection of economical algorithms for multi-stage microenvironment detection, to cut back operating time and also the sorts of sensors, particularly actuators, area unit rigorously are chosen for the aim of effectiveness and non-intrusiveness. As an example, sensible Smart Android App won't trigger vibrator or speaker once a wise phone is carried by its user.

Local Placement Recognition: This module determines daily on-body phone placements like in-hand, in-packet, in-bag, etc. sensible Smart Android App provides a straightforward nonetheless effective classification theme with light weight and mechanical phenomenon sensors. Specifically, the system 1 st detects whether or not the phone is in hand by referring close illuminative conditions round the phone. If not, then, sensible Smart Android App characterizes the distinctive moving patterns of phones in numerous native placements with in-built measuring instrument, by exploiting a Dynamic Time warp (DTW) based mostly statistic matching theme to acknowledge the particular native placement. Phone Interaction Detection: This module identifies whether or not the user is truly victimization the phone, like browsing. Though such interaction typically happens once the phone is in- hand, the phone interaction detection module emphasizes additional on the linguistics perspective. Smart Android App exploits common screen-lock on sensible phones and method transition on OS to spot whether or not the user is truly interacting together with his phone.

Backing Material Detection: This module differentiates hard/soft material via good phone-generated vibration patterns. Good automaton App focuses on 2 aspects of the vibration patterns: 1) the phone's mechanical motion and 2) the physical science options, which might be captured by embedded measuring system and electro-acoustic transducer, severally. To the current finish, Smart automaton App extracts a series of light-weight options from acceleration/acoustic traces in each time and frequency domain, and classifies backing materials like animal skin chair, wood table or glass table. Placement of the phone is major consideration during this phenomena, which is detected with the help of sensors. The key insights calculate twofold. Once carried by a user, the phone is usually placed in either semi-closed/open environments like in-hand, or closed environments like inpocket and in-bag. The extent of covering results in totally different illuminative conditions for the phone, which might be captured by its inherent camera. Totally different native surroundings supply distinctive spatial degree of freedom, that is exaggerated once the user is moving. As an example, a phone is probably going to expertise fiercer movements once place in pants than within a purse.

Intuitively, the 'in-hand' state differs from on-body placements in this the phone isn't utterly lined by close objects. Though the front-mounted proximity detector will understand sheltering ahead, the phone is unaware of that backwards. therefore with proximity detector alone, it's seemingly to miss some 'in-hand' cases, e.g., once the user is creating a call together with his ear covers the side of the phone. Additionally, usage of the back-mounted camera for proximity perception backwards has also considered. The rationale is that the global contrast of a photo taken in a closed environment is usually low, which is reflected in the grayscale histogram of the photo.

Hence, in this projected system it combines the information from multiple sensors and makes applications supported them.

\section{EXISTING SYSTEM}

Presently there is application which is based on single sensor. The application was made by making use of data broadcasted by sensors. But these applications had several drawbacks like it consumes more battery as it has to run continuously. Also there were no supporting applications for battery saving purpose. There are some drawbacks in the existing system which are ,

- Requires much energy for sensing.

- It takes long time to sense the application.

- Security is compromised.

- Accuracy is not achieved.

- Consumption of memory.

\section{PROPOSED SYSTEM}

A micro-environment sensing platform that mechanically records sensing element knowledge and characterizes the micro-environment of Smartphone's in terms of application is projected. The platform runs as a daemon method on a sensible phone and provides finer-grained setting data to higher layer applications via programming interfaces. This platform run in middleware stage and supply knowledge that is captured by varied sensors to the appliance that is used in the application via programming interface 


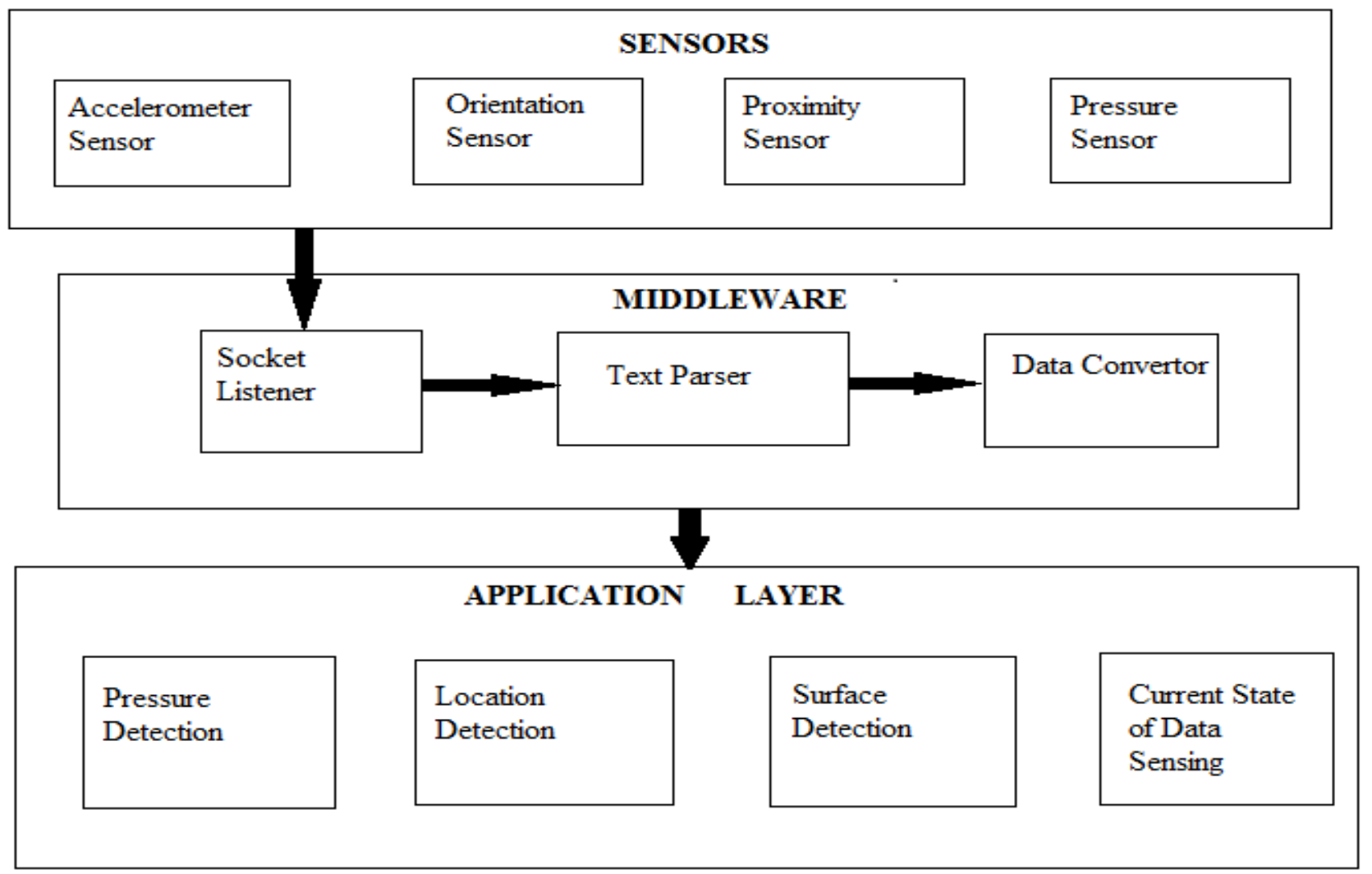

Fig 1: Proposed System Architecture

This application may be a unified framework covering the most important cases of phone usage, placement, attitude, and interaction in sensible uses with difficult user habits. As a semi permanent running middleware, application considers each energy consumption and user relationship. Therefore an application on Android OS is developed which systematically evaluates its performance with data collected. The preliminary results show that an application achieves low energy cost, rapid system deployment, and competitive sensing accuracy.

\subsection{Advantages of proposed system}

- Smart Android App consumes negligible energy of below 5 percent on average for most sampling periods. With time passing by, the cumulative energy consumption increases gradually and finally ends up with 11.2 percent.

- As there is usage of multiple sensors in this project, introducing different parsing techniques for each sensor on the same time battery optimization is also cared.

- The application will result in Competitive MicroEnvironment Sensing Accuracy.

- GPS which helps to estimate user's coarse-grained macro-environment but, Smart Android App deduces phone's fine-grained micro-environment.

- It serves as a light-weighted middleware for upper layer applications.

\section{CONCLUSION}

Thus designing an Application which senses the MicroEnvironment via collaboration among built in sensors and achieving data or information from the environment is proven successful with its implementation. Here clubbing various sensors in this application of whose result achieves low Energy Cost and Competitive Micro-Environment Sensing Accuracy has also proven useful. The platform automatically collects sensor hints and characterizes the immediate surroundings of smartphones providing environment information to upper layer applications.

Thus in this modern period of use of smartphones usage of these various sensors in them, grabbing the environmental changes will prove very much useful for future purpose. Taking care of many aspects it is useful and is needed in today's generation of smartphones, as providing a set of features in a single smartphone without extra usage of data space and battery usage.

\section{REFERENCES}

[1] J. Yang, S. Sdhom, G. Chandrasekaran, T. Vu, H. Liu, N.Cecan, Y. Chen, M. Gruteser, and R. Martin, "Detecting Driver Phone Use Leveraging Car Speakers," Proc. ACM MOBICOM, 2011.

[2] S. Nath, "ACE: Exploiting Correlation for EnergyEfficient and Continuous Context Sensing," Proc. 10th Int'l Conf. Mobile Systems, Applications, and Services (MobiSys '12), 2012.

[3] T. Yan, D. Chu, D. Ganesan, A. Kansal, and J. Liu, "Fast App Launching for Mobile Devices Using Predictive User Context" Proc. 10th Int'l Conf. Mobile Systems, Applications, and Services(MobiSys '12), 2012.

[4] C. Qin, X. Bao, R.R. Choudhury, and S. Nelakuditi, "Tagsense:A Smartphone-Based Approach to Automatic Image Tagging "Proc. Ninth Int'l Conf. Mobile Systems, Applications, and Services(MobiSys '11), 2011. 
[5] H. Lu, W. Pan, N.D. Lane, T. Choudhury, and A.T. Campbell," Sound sense: Scalable Sound Sensing for People-Centric Applications on Mobile Phones," Proc. Seventh Int'l Conf. Mobile Systems ,Applications, and Services (MobiSys '09), 2009.

[6] H. Lu, J. Yang, Z. Liu, N.D. Lane, T. Choudhury, and A.T.Campbell,"The Jigsaw Continuous Sensing Engine for Mobile Phone Applications," Proc. Eighth ACM Conf. Embedded Networked Sensor Systems (SenSys '10), 2010.
[7] M. Azizyan, I. Constandache, and R. Choudhury, "Surround Sense :mobile Phone Localization via Ambience Fingerprinting," Proc.ACMMOBICOM, 2009.

[8] A. Rai, K. Chintalapudi, V. Padmanabhan, and R. Sen, "Zee: Zero-Effort Crowd sourcing for Indoor Localization," Proc. ACM MOBICOM,2012.

[9] P. Zhou, Y. Zheng, Z. Li, M. Li, and G. Shen, "IODetector: A Generic Service for Indoor Outdoor Detection," Proc. 10th ACM Conf. Embedded Networked Sensor Systems (SenSys '12), 2012. 